\title{
Recuperación elástica
}

\author{
Elastic Recovery \\ Miguel A. Flores-Rentería ${ }^{a}$, Martín Ortiz-Domínguez $^{b}$, Arturo Cruz-Avilés $^{c}$
}

\begin{abstract}
:
The process of forming sheet metal is one of the most important, consists, by means of a tooling deforming a sheet metal until taking the shape of a certain geometry, forging, extrusion, bending are some of the forming processes, it is performed in hot or cold, both are characterized by a great deformation of the workpiece. At the end of this process there is a difference between the final geometry obtained and the desired what can be considered as manufacturing defects, is usually known as elastic recovery, is a consequence of the properties of the material, the working tools required to give the desired shape, etc. There are different alternatives to correct it such as the die and punch design to obtain the desired geometry considering elastic recovery, or modification of the mechanical properties of the material. In this work a finite element model is developed to observe the behavior of elastic recovery by increasing the magnitude of young's module in its solution an ANSYS software was used, in the results it is observed that the smaller its magnitude, the greater the elastic recovery of the material.
\end{abstract}

\section{Keywords:}

Elastic recovery, Young modulus, Forming process

\begin{abstract}
Resumen:
El proceso de formado de chapa metálica es uno de los más importante, consiste en, por medio de un herramental deformar una chapa metálica hasta tomar la forma de una determinada geometría, la forjar, la extrusión, el doblado son algunos de los procesos de formado, se realiza en caliente o frío, ambos se caracterizan por una gran deformación de la pieza de trabajo. Al final de este proceso se presenta una diferencia entre la geometría final obtenida y la deseada lo que se puede considerar como defectos de fabricación, generalmente es conocida como recuperación elástica, es consecuencia de las propiedades del material, de los herramentales de trabajo requeridos para dar la forma deseada, etc. Existe diferentes alternativas para corregirla como lo es el diseño de la matriz y el punzón para obtener la geometría deseada considerando la recuperación elástica, o la modificación de las propiedades mecánicas del material. En este trabajo se desarrolla un modelo de elementos finitos para observar el comportamiento de la recuperación elástica al increment ar la magnitud del módulo de Young en su solución se empleó el software ANSYS, en los resultados se observa que mientras menor es su magnitud, mayor es la recuperación elástica del material.
\end{abstract}

\section{Palabras Clave:}

Recuperación elástica, módulo de Young, Proceso de formado

\footnotetext{
Autor de Correspondencia, Universidad Autónoma del Estado de Hidalgo, https://orcid.org/0000-0001-8018-4708, Email: miguel_flores10233@uaeh.edu.mx

b Universidad Autónoma del Estado de Hidalgo, https://orcid.org/0000-0003-4475-9804, Email: martin_ortiz@uaeh.edu.mx

c Universidad Autónoma del Estado de Hidalgo, https://orcid.org/0000-0003-0455-1646,Email: arturo_cruz8085@uaeh.edu.mx
} 


\section{Introducción}

En la industria automotriz y aeronáutica uno de los procesos de manufactura más importantes es el formado de lámina, asociado a este se presenta la recuperación elástica, la cual representa un cambio geométrico de una pieza al final del proceso, en este sentido el determinar con anticipación su tamaño y dirección es esencial. La recuperación elástica se presenta principalmente cuando un metal es doblado, después de retirar la carga este intenta recuperara su forma original, afectando la precisión de la pieza (ver figura 1),

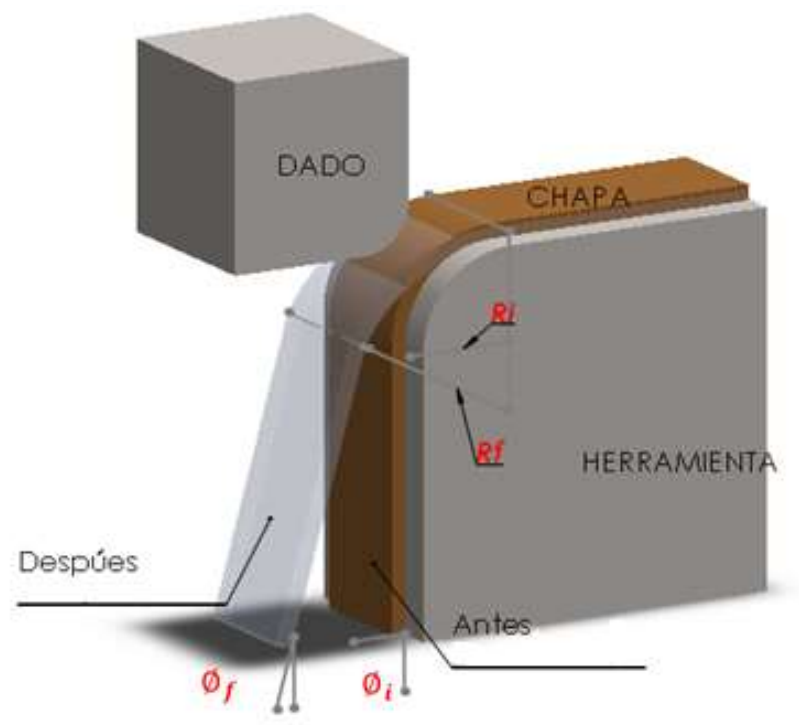

Figura 1. Recuperación elástica

Durante este proceso a medida que el material se dobla la región interior de la curva se compacta mientras que la exterior se extiende.

El estado de deformaciones que se crea conduce a la formación de esfuerzos residuales, después de la liberación de la carga estos esfuerzos se manifiestan como una recuperación elástica que a su vez se expresa por cambios geométricos no deseados, así mismo la densidad atómica es mayor dentro de la curva, por lo que la fuerza de compresión es mayor que la elástica, por consecuencia se dañarán las áreas externas de la pieza antes de que se distorsione el área interior (ver figura 2). La recuperación elástica se puede definir en términos de una relación de ángulos, entre el producido y el deseado. Se han realizado varios trabajos para analizarla, analítica, experimental y numéricamente con diferentes formas y parámetros del material (Kalpakjian \& Schmid, 2014), (Hosford \& Caddell, 2007).

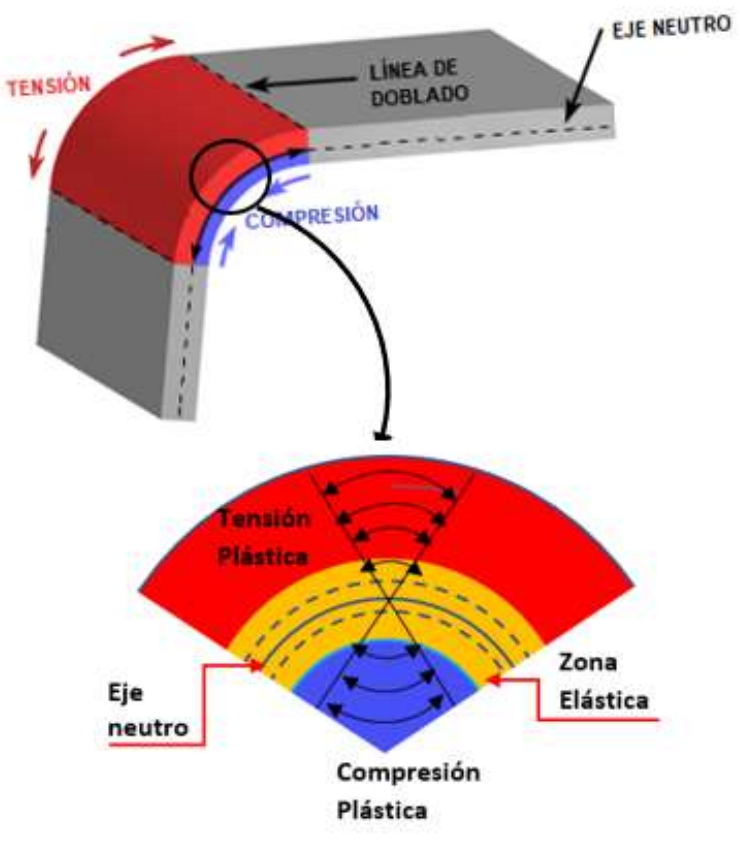

Figura 2. Tensión y compresión en la recuperación elástica,

La calidad del formado de chapa metálica depende del diseño adecuado de las herramientas, la elección del material, la fuerza de sujeción, lubricación, entre otros parámetros del proceso, en este sentido, se necesita un amplio conocimiento sobre la influencia de estos parámetros. Para establecer esta base de conocimiento, se utilizan pruebas experimentales o simulaciones numéricas. La simulación es una herramienta poderosa que permite modificar cualquiera de los parámetros, repetir la prueba tantas veces como sea necesario y predecir la recuperación elástica.

La recuperación elástica no se puede eliminar, sin embrago existen métodos para reducirla como lo es el diseño adecuado de las matrices, la selección adecuada de los parámetros y el uso de métodos avanzados de predicción de la forma final por medio del método de elementos finitos (FEM), actualmente este es la principal técnica utilizada para simular procesos de conformado de chapa metálica y determinar la distribución de esfuerzos 
y deformaciones, así como de las fuerzas de formando y posibles ubicaciones de los defectos.

\section{Formado de chapa metálica}

En el proceso de formado de chapa metálica cada deformación plástica es seguida por una recuperación elástica. La deformación permanente $\left(\varepsilon_{t}\right)$ se expresa como la diferencia entre las deformaciones plástico $\left(\varepsilon_{p}\right)$ y elásticas $\varepsilon_{e}$ :

$$
\varepsilon_{t}=\varepsilon_{p}-\varepsilon_{e}
$$

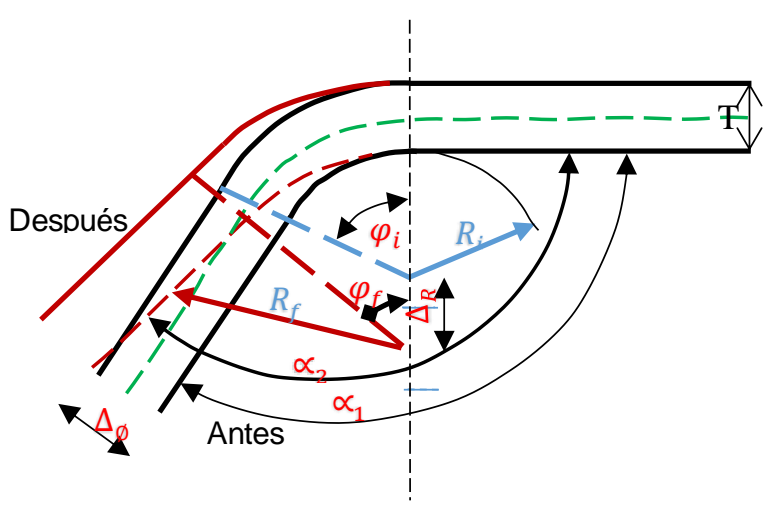

Figura 3. Radios iniciales y finales

En la figura 3 las dimensiones iniciales y finales del radio de doblez se representa por $R i$ y $R f$, los ángulos de doblado por $\varphi_{i}=180^{\circ}-\alpha_{1}$ y $\varphi_{f}=180^{\circ}-\alpha_{2}$ así como los perfiles del ángulo $\alpha_{1}$ y $\alpha_{1}$ respectivamente.

Después de la recuperación del material se obtiene que $\varphi_{f}<\varphi_{i}$ y $R_{f}>R_{i}$ lo cual implica que el ángulo final es pequeño y el radio final grande.

Un valor que caracteriza a la recuperación elástica es el factor $\mathrm{K},\left(K=\frac{R_{i}}{R_{f}}\right)$ el cual se define considerando que la línea neu tra $L_{n}$ es la misma antes y después del doblado, con esto se obtiene la siguiente relación ver figura 3.

$$
\begin{gathered}
L_{n}=\left(R_{i}+\frac{T}{2}\right) \varphi_{i}=\left(R_{f}+\frac{T}{2}\right) \varphi_{f} \\
K_{S}=\frac{R_{i}+\frac{T}{2}}{R_{f}+\frac{T}{2}}=\frac{\frac{2 R_{i}}{T}+1}{\frac{2 R_{f}}{T}+1}=\frac{\varphi_{f}}{\varphi_{i}}=\frac{180^{\circ}-\propto_{2}}{180^{\circ}-\propto_{1}}
\end{gathered}
$$

El factor $\mathrm{K}$ depende únicamente de la relación $R / T$. Cuando $K=1$ indica que no hay recuperación elástica y cuando $\mathrm{K}=0$ indica una recuperación elástica completa. Para su estimación se ha desarrollado la siguiente expresión en términos los radios iniciales y finales $R_{i}$ y $R_{t}$ es;

En donde

$$
\frac{R_{i}}{R_{f}}=4\left(\frac{R_{i *}(Y S)}{E * T}\right)^{3}-3\left(\frac{R_{i}(Y S)}{E T}\right)+1
$$

$R_{i}$ Radio inicial

$R_{f}$ Radio final

YS Límite de fluencia

E Módulo elástico

$T$ Espesor de la placa

\section{Simulación de la recuperación elástica con método de elementos finitos}

A través del método de elementos finitos se ha podido determinar el comportamiento de los diferentes parámetros involucrados en la recuperación elástica, por ejemplo, los efectos de; las fuerzas de sujeción, de la geometría de los herramentales, de la dureza de los materiales etc. En la literatura se encuentra reportada una cantidad considerable de investigaciones sobre el formado de chapa metálica y recuperación elástica, así como de su modelado, en los cuales se ha analizado, por ejemplo, el comportamiento de la recuperación elásticay la predicción en aceros de alta resistencia tanto numérica como experimentalmente (Andersson, 2007), así como el efecto de la fricción variando los parámetros de los herramentales, en donde se demostró que un coeficiente de fricción alto incrementa la tensión aplicada a la chapa metálica, lo que disminuye el momento requerido para realizar una curvatura y reduce la variación de esfuerzos en el espesor de la placa y por lo tanto la recuperación elástica es menor (Kim \& Koç, 2008), se ha estudiado los efectos que tienen los parámetros geométricos y el endurecimiento del materiales sobre la recuperación elástica en este trabajo se usó una red neuronal artificial (Kazan \& Firat M, 2009), para entender el comportamiento de la recuperación elástica se han desarrollado simulaciones de diferentes materiales tales como alu minio, acero inoxidable y acero bajo en carbono de varios grados y espesores (Ruszkiewicz, Grimm, \& Ragai, 2017), la aplicación del método de elementos finitos ha mostrado que se tiene diferentes gradientes de tensiones a lo largo del espesor de chapa y que esa línea de tensiones es de suma importancia para predecir la recuperación elástica. 


\section{Modelo de elementos finitos}

Se ha desarrollado un modelo en 3D para simular el proceso de formado y con la finalidad de investigar el efecto del módulo de Young sobre la recuperación elástica en chapa metálica de acero.

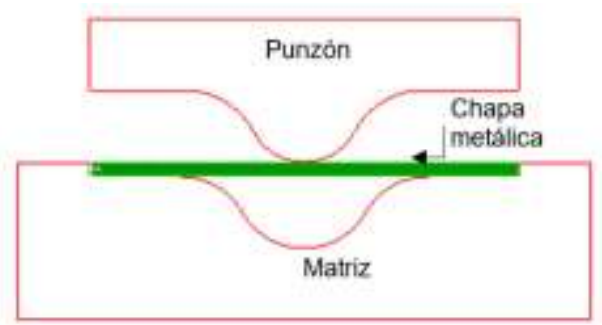

Figura 4. Modelo de elementos finitos

El proceso se realizó en dos pasos; en el primer se simuló el proceso de formado, mientras que el segundo se liberó la tensión. El material que se analiza es chapa de acero usando un modelo de plasticidad no lineal variando las siguientes propiedades; módulo de Young 2, 2,5 y 3 Gpa, respectivamente.

En un proceso de formado la matriz es una herramienta que permanece fija durante todo el proceso, el pisador es la herramienta que ejerce una fuerza constante sobre la placa para evitar problemas de arrugamiento, el punzón es el encargo de deformar el material por medio de un desplazamiento, para esto se usó la curva mostrada en la figura 5 la cual inicia y termina con una velocidad cero, esto con el objeto de disminuir los efectos dinámicos.

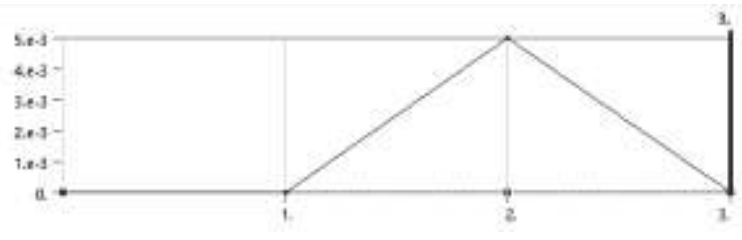

Figura 5. Curva de desplazamiento.

El contacto entre elementos es asimétrico del tipo friccional, con coeficiente de fricción de 0.1 , está basado en el método de Lagrangiano Aumentado el cual hace uso de la integración de Gauss para determinar puntos a lo largo de los segmentos en contacto, considera la penalización lo que permite la penetración de contacto controlada, se utiliza un factor de rigidez de 0.1 .

Las simulaciones son del tipo dinámico explícito, en las cuales se están considerando el incremento de la energía cinética causada por la velocidad virtual de la herramienta.

Se empleo en la chapa un mallado del tipo Uniform Quad Mesh Method el cual permite que la malla generada tenga una estructura y densidad uniformes, utiliza elementos Quad8. Se sabe que un mallado fino ofrece una mayor densidad de elementos y con esto una mejor descripción de la geometría, sin embargo, esto significa un mayor número de elementos y por consiguiente un mayor tiempo de solución de cómputo. El tamaño de elementos utilizados en el mallado para evaluar recuperación elástica es de $1.5 \mathrm{~mm}$.

\section{Resultados}

Se han obtenido los esfuerzos equivalentes máximos, los cuales utilizan la teoría de Von-Mises para determinar un esfuerzo equivalente a partir de una combinación particular de esfuerzos principales, esto permite entender el comportamiento de la estructura y, en última instancia, la probabilidad de fallo o puntos críticos. El esfuerzo equivalente máximo también permite evalúa el factor de seguridad para materiales dúctiles, en el proceso de formado se requiere que el material se someta a deformación plástica sin que el material falle en este punto.

En la figura 6 se muestran una secuencia del proceso, en la tabla 1 se presentan los esfuerzos von Mises correspondientes a cada etapa, se observa que el esfuerzo máximo obtenido es de $427 \mathrm{Mpa}$ los cuales no exceden el límite de tensión final (200 Gpa), lo cual indica que el material en particular y el diseño del punzón es, aparentemente, una alternativa viable para la fabricación de la pieza diseñada.

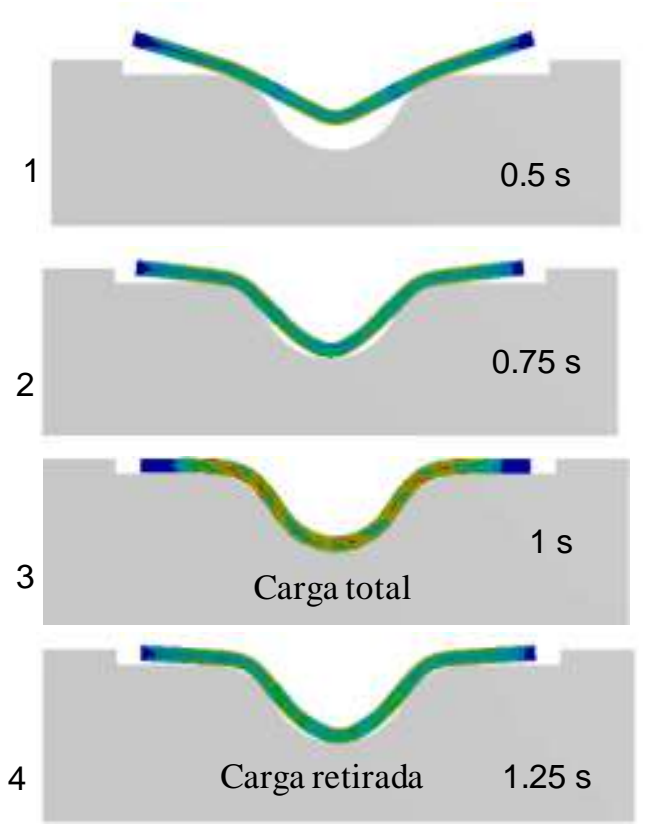

Figura 6. Etapas de la simulación computacional. 


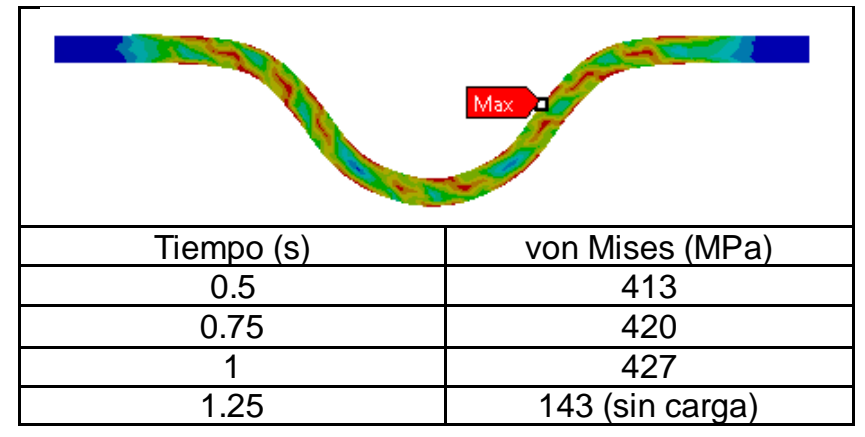

Tabla 1

La figura 7 es una amplificación de la etapa 4 después de retirar la carga, obsérvese como la pieza terminada no tiene la forma de la matriz.

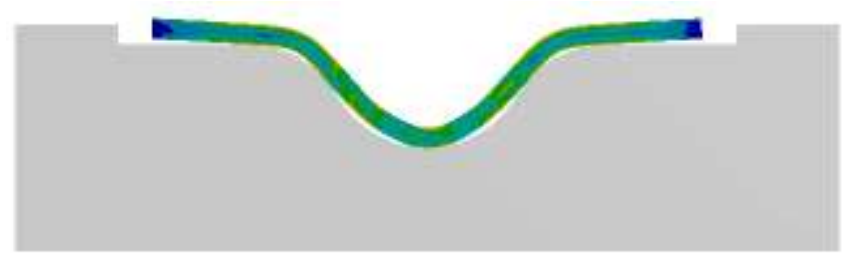

Figura 7

El producto final tiende a recuperar su forma, este efecto es conocido como recuperación elástica, dependiendo de la funcionalidad de la pieza y de las tolerancias de diseño puede o no ser permitida esta deformación.

Una de las grandes ventajas de la simulación computacional es que se puede variar cualquier parámetro y observar su comportamiento, en este estudio se ha modificado el módulo de Young del material de 2, 2.5, y 3 Gpa para observar su efecto en la recuperación elástica, los resultados se aprecian en las figuras siguientes.

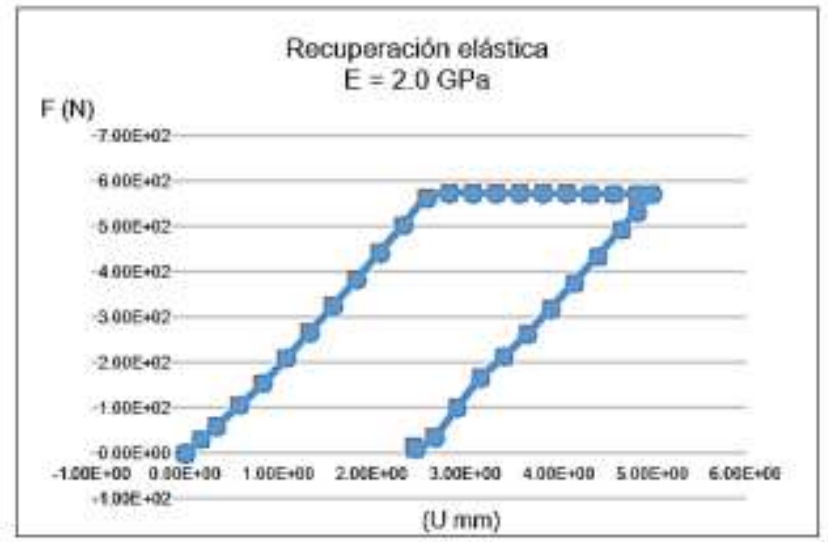

Figura 8 (a)

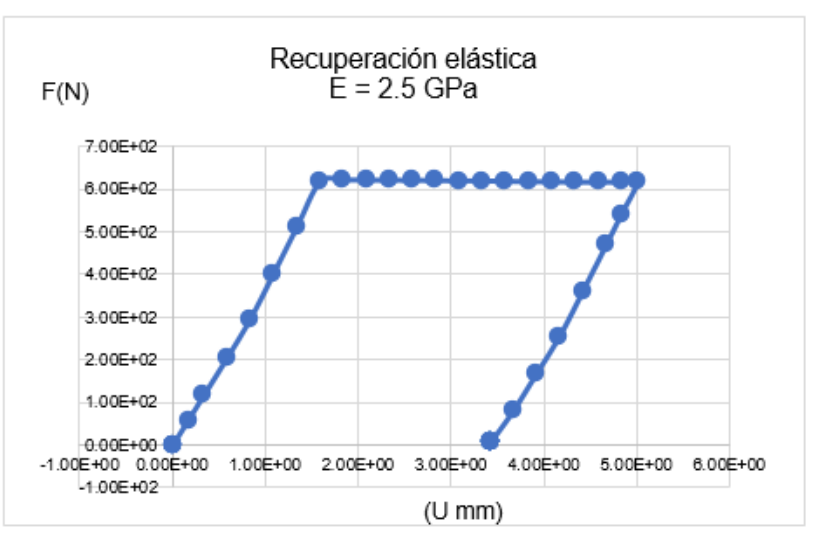

Figura 8 (b)

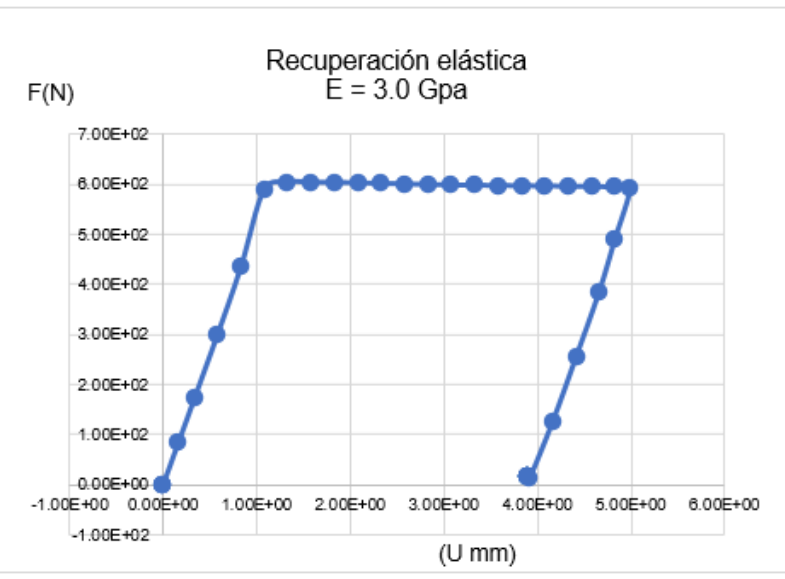

Figura 8 (c)

En las figuras $8(a, b, c)$ se muestran las gráficas de carga vs deformación de la variación del módulo de Young, de la cual se determina qué; cuanto menor sea el módulo de Young mayor será la recuperación elástica, cuanto mayor sea el límite elástico del material mayor será la recuperación elástica. El endurecimiento del material por deformación o por proceso también tiene consecuencias, cuanto más se endurezca el material la recuperación elástica será mayor.

La simulación computacional es una importante alternativa para optimizar el diseño de troqueles, su aplicación reduce las correcciones de los mismos du rante la puesta en operación, durante el proceso de diseño de estos herramentales se necesita una predicción numérica precisa del proceso que permita disminuir el tiempo de desarrollo y el costo del troquel.

\section{Conclusión}

El fenómeno de la recuperación elástica está relacionado con el estado tensional que se genera en la chapa metálica. Los parámetros que afectan a dicho estado 
tensional, pueden ser tanto del material como lo es la elasticidad, el criterio de fluencia y el endurecimiento o parámetros del proceso como, el efecto tribológico, la fuerza de pisado, los tratamientos térmicos entre otros. En este sentido la modelización y caracterización de los parámetros es importante. Dependiendo de la geometría del componente, un parámetro en específico será de mayor peso que otro, en algunos casos es suficiente con aumentar la presión del pisador sobre la chapa para reducir la recuperación elástica.

Para poder caracterizar la recuperación elástica de un material, es indispensable elegir la simulación computacional que mejor reproduzca las condiciones tensionales que se dan en el proceso de formado. Estas pueden ser simulaciones no restringidas en las cuales el herramental está compuesto por un punzón y una matriz o pueden ser restringidas en los cuales el herramental dispone de un pisador además del punzón y de la matriz. El aumentar la magnitud del módulo de Young es una alternativa para reducir la recuperación elástica, esto implica la sustitución del material, que en algunos casos puede conducir a incremento en el costo del producto, en otras situaciones se puede modificarse la geometría del punzón y la matriz de acuerdo con los resultados obtenidos en la simulación computacional, es importante reducir los esfuerzos residuales generados por el proceso de deformación lo cual se puede lograr incrementado el tiempo de compresión al final de la carrera de deformación del punzón.

Las variables que intervienen en un proceso de formado de chapa metálica son varias, principalmente los radios de doblado en las matrices, punzones y la geometría deseada por lo que la optimización del proceso es necesaria para obtener la mejor solución de acuerdo con el mejor veneficio entre la producción, el costo y la calidad.

\section{Referencias}

Andersson, A. (2007). Numerical and experimentalevaluation of springback in advanced high strength steel. . Journal of Materials Engineering and Performance, 301-307.

Hosford, W., \& Caddell, R. (2007). Metal forming mechanics and metallurgy. Michigan: Cambridge.

Kalpakjian, S., \& Schmid, S. (2014). Manufacturing engineering and technology. Singapore: Prentice Hall.

Kazan, R., \& Firat M, M. (2009). Prediction of springback in wipebending process of sheet metalusing neural network. . Materials \& Design, 418 - 423.

Kim, H., \& Koç, M. (2008). Numerical investigations on springback characteristics of aluminum sheet metalalloys in warm forming conditions. . Journal of Materials Processing Technology, 370 - 383.

Ruszkiewicz, B., Grimm, I., \& Ragai, L. (2017). A Review of ElectricallyAssisted Manufacturing with Emphasis on Modeling and Understanding of the Electroplastic Effect. Journal of Manufacturing Science and Engineering, 110-121. 